\title{
Analysis of Piezoelectric Sensors in Adulteration of Bovine Milk Using the Chromatic Technique ${ }^{\dagger}$
}

\author{
Marcos Messias dos Santos Junior 1,*, José Alfredo Covolan Ulson 1, \\ Bruno Albuquerque de Castro ${ }^{1}$, Jorge Alfredo Ardila-Rey ${ }^{2}$, Fernando de Souza Campos ${ }^{1}$ and \\ Laura Teófilo Ferreira ${ }^{3}$ \\ 1 São Paulo State University (UNESP), School of Engineering, Bauru, Department of Electrical Engineering, \\ 17033-360 Bauru-SP, Brazil; alfredo.ulson@unesp.br (J.A.C.U.); bruno.castro@unesp.br (B.A.d.C.); \\ fernando.casmpos@unesp.br (F.d.S.C.) \\ 2 Department of Electrical Engineering, Universidad Técnica Federico Santa María, Av. Vicuña Mackenna \\ 3939, Santiago de Chile 8940000, Chile; jorge.ardila@usm.cl \\ 3 Department of Chemistry, São Paulo State University, Av. Eng. Luiz Edmundo Carrijo Coube 14-01, \\ 17033-360 Bauru, Brazil; laura.teofilo@unesp.br \\ * Correspondence: marcos.messias@unesp.br; Tel.: +55-11-98538-1298 \\ + Presented at the 5th International Electronic Conference on Sensors and Applications, 15-30 November \\ 2018; Available online: https://ecsa-5.sciforum.net.
}

Published: 14 November 2018

\begin{abstract}
Sensors applied in the food industry are important tools for quality control. Current analyses checking adulteration in milk are expensive and time consuming, because the samples need to be evaluated in a laboratory environment. Thus, is important to develop methodologies and sensors to monitor milk production. A common type of fraud is performed adding substances such as sodium hydroxide in order to increase the shelf life of milk. In this study, we propose to use lowcost piezoelectric diaphragms transducers to implement a methodology that identifies milk adulteration using the mechanical waves propagation method (vibration and acoustic emission). Two piezoelectric diaphragms were used, the first was excited by a chirp signal with $1 \mathrm{~V}$ of amplitude and a frequency band since 0 to $65 \mathrm{kHz}$ with $2 \mathrm{~Hz}$ of step, and concomitantly acquired the response signal of the second sensor installed in the opposite side of the actuator with a rate of $250 \mathrm{kHz}$. After acquiring the data, these were processed using the chromatic technique, which extracts three features: energy, average band and equivalent bandwidth, in order to classify the raw and the contaminated milk through clustering. The experimental results indicated that the methodology can differentiate between raw and contaminated milk with $1 \%$ of sodium hydroxide. Therefore, the results reported in this study indicate that low-cost piezoelectric diaphragms are promising for liquids quality control.
\end{abstract}

Keywords: piezoelectric diaphragms; low-cost; bovine milk adulteration; chromatic technique

\section{Introduction}

The development of quality control tools applied to the food industry is essential for preventing some issues such as food adulteration. Adulterated food is dangerous for health because it may contain several toxic substances which can hinder the normal development of the human body or even lead to serious disorders and sickness [1-3]. Therefore, nowadays, both science and industry have sought to develop sensors and devices aiming to promote the correct quality control of food diagnosis conditions, in order to promote human health and consumption safety. One of the most commonly adulterated foods is milk. A common type of fraud is accomplished in order to increase milk shelf life by adding substances such as water, sodium hydroxide $(\mathrm{NaOH})$, peroxide hydrogen 
$\left(\mathrm{H}_{2} \mathrm{O}_{2}\right)$, etc. [1-3]. As an example, the effects of sodium hydroxide on human health can be a stomachburning sensation, nausea and vomiting [2].

Most milk adulteration measurements are expensive and time consuming, as the milk samples need to be taken to the dairy laboratories for testing. Therefore, currently, the development of low cost, rapid and reliable sensors and systems which aim to detect milk adulteration is essential to guarantee the quality level of human health, safety and the quality of industrial production [3]. In order to contribute in this issue, in this study, we propose to implement a methodology to identify milk adulteration by acoustic emission propagation method using low-cost piezoelectric diaphragms. Acoustic waves will be propagated in the milk and the acoustic signals will be processed by the chromatic signal processing technique (CT) [4]. This promising digital signal processing can be used to achieve a patter recognizing or cluster data and it will be applied to separate samples of pure and contaminated milk. As an initial study, we use sodium hydroxide $1 \%$, as a chemical adulteration in a sample of pure milk. This type of contamination is commonly accomplished in industry, according to reference [2]. The results indicated that the proposed method has great applicability in the identification of milk adulteration.

The outline of this article is as follows: Section 1.1 presents the basics concepts of acoustic emission by piezoelectric transducers. Section 1.3 presents the concepts of chromatic cluster technique and the experimental setup is described in Section 2. In Section 3, the results are discussed and the conclusion of this paper is presented in Section 4.

\subsection{Piezoelectric Sensors and Acoustic Emission (AE)}

The piezoelectric transducers can operate both as sensors and actuators due to the piezoelectric effect [5-8]. The piezoelectric effect occurs in materials that, when subjected to a mechanical stress, produce an output voltage by the direct effect. The reverse effect also occurs: by applying an electric voltage to the piezoelectric materials, a mechanical deformation is produced [5-8]. Due the piezoelectric effect, the transducer can be configured as an actuator, and vibration or ultrasound waves can be emitted by applying a voltage in a determined frequency range. Besides that, it also can be configured as a sensor, since the piezoelectric is sensitivity to vibration and acoustic waves.

Figure 1 presented the transducer applied in this work, which is commonly applied in structure monitoring [7,9] and in partial discharge diagnosis [6]. This type has a ceramic disk of $25.0 \mathrm{~mm} \times 0.23$ $\mathrm{mm}$ and a brass plate of $35.0 \mathrm{~mm} \times 0.30 \mathrm{~mm}[10]$.
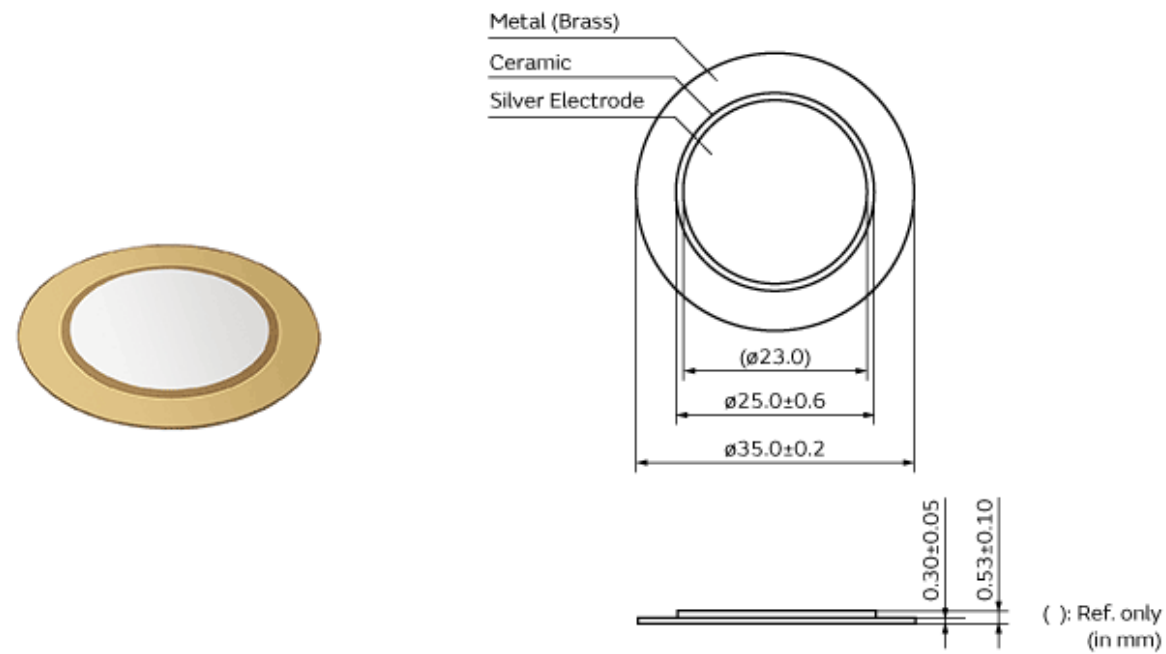

Figure 1. Murata piezoelectric sensor 7BB-35-3 used in the experiment [10].

Acoustic wave propagation can change according to the fluid properties [11]. In this work we use two piezoelectric transducers, one operating as the actuator and the other operating as a receiver sensor (pitch-catch mode) [12]. The emitter had the function of generating the mechanical waves in a 
sample of milk and the adulteration detection will be accomplished by processing the acoustic signals captured by the receptor, since the AE propagation can change with the material alters or fluid contamination. As explained in the follow section and in the experimental setup, the chromatic technique is used to differentiate the raw and contaminated milk by $1 \%$ of $\mathrm{NaOH}$.

\subsection{Chromatic Technique (CT)}

The chromatic technique is a signal processing approach which aims to provide a series of parameters that facilitate the extraction of information from a complex group of signals whose characteristics cannot be easily identified [13-15]. It has been demonstrated that this technique also enables, for some types of applications, the identification and the correlation of the parameters that define the signals, determining if a group of signals can be identifiable from a chromatic point of view [9-11]. According to this technique, the signal $f(t)$ classification is carried out by means of three signal principal parameters: energy $(E)$, average band $\left(\omega_{c}\right)$ and equivalent bandwidth $\left(B_{A v}\right)$, as follows:

$$
\begin{gathered}
E=\frac{1}{2 \pi} \sum_{i=1}^{k} F_{i}, \\
\omega_{c}=\frac{\sum_{i=1}^{k} \omega_{i} F_{i}^{2}}{2 \pi E_{b}}, \\
B=\sqrt{\frac{1}{E_{b}} \sum_{i=1}^{k}\left(\omega_{i}-\omega_{c}\right)^{2} F_{i}^{2}},
\end{gathered}
$$

where $F_{i}$ and $\omega_{i}$ is the Fourier transform and the angular frequency of a signal $f[n]$ in discrete time.

In this work, the CT was accomplished in order to cluster 200 samples of milk, in which 100 is regarded as raw milk and another 100 samples were regarded as adulterated milk.

\section{Experimental Setup}

As the objective is to perform a milk adulteration detection, the sensor device was built by applying two piezoelectric on each end of a test tube, as shown in Figure 2.

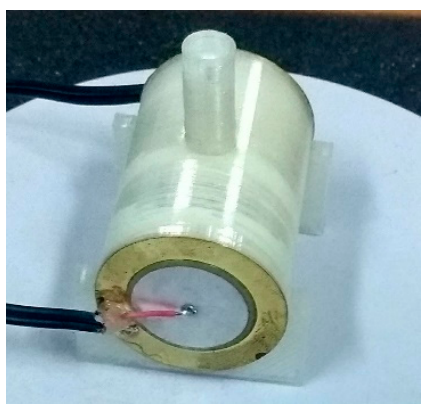

(a)

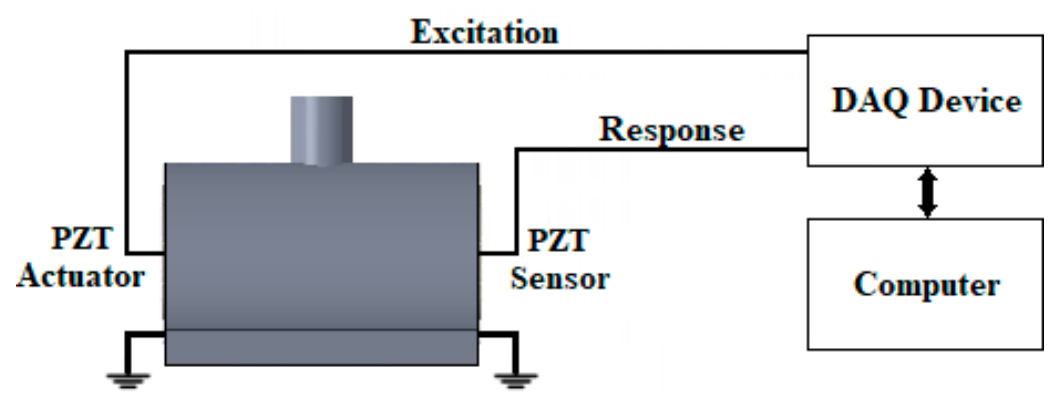

(b)

Figure 2. Experimental Setup (a) and sensor device (b).

The setup was performed using a NI USB-6211 data acquisition (DAQ) device with a sampling rate of $250 \mathrm{kHz}$. The actuator was excited by a signal chirp whose amplitude was $1 \mathrm{~V}$ and whose frequency range was from $0 \mathrm{kHz}$ to $65 \mathrm{kHz}$ with a step of $2 \mathrm{~Hz}$. The signal of the receptor, installed in the opposite side of the actuator, was acquired with a sample rate of $250 \mathrm{kHz}$.

The receptor signal was processed by chromatic technique. Two hundred samples of milk, of which one hundred were adulterated with $\mathrm{NaOH}$ and the others one hundred samples were a raw milk, were inserted in a test tube. This setup produced 200 signals that were processed by the CT. The results are presented in the next section. 


\section{Results and Discussion}

Figure 3 shows the results obtained by applying the chromatic signal processing technique. It was used as a clustering and separation approach in order to promote the correct milk diagnosis condition.

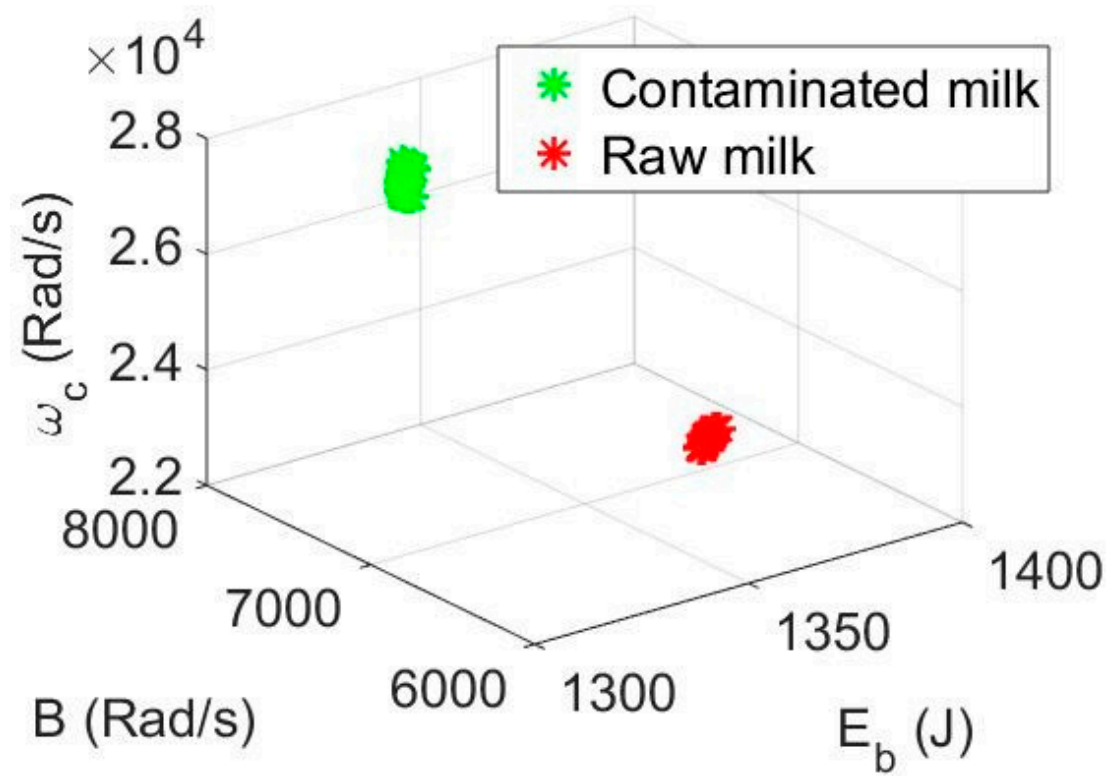

Figure 3. 3D classification map of the chromatic technique.

As observed in the result presented, the CT performed a correct feature extraction for milk condition; the color maps formed by the 100 data points of raw milk are totally separated from the 100 data points of contaminated milk. Table 1 presented the average of each CT parameter for raw and adulterated milk.

Table 1. Average of CT parameters.

\begin{tabular}{ccc}
\hline Feature & Raw Milk & Adulterated Milk \\
\hline Energy $\mathrm{Eb}(\mathrm{J})$ & 1337 & 1365 \\
Average band B $(\mathrm{Rad} / \mathrm{s})$ & 7746 & 6661 \\
Equivalent bandwidth $\omega \mathrm{c}(\mathrm{Rad} / \mathrm{s})$ & 26,862 & 23,262 \\
\hline
\end{tabular}

As observed, the average energy was $2 \%$ higher for adulterated milk in relation to the raw milk. However, the average band and equivalent bandwidth decreases, respectively $14 \%$ and $13.4 \%$ for adulterated milk in relation to the pure milk.

In order to study the relevance of each parameter to cluster the milk condition and perform the correct diagnosis of adulteration, the Figure $4 \mathrm{a}-\mathrm{c}$ presented the 2D maps of the chromatic parameters.

Regarding these results, it can be concluded that all parameters have a significant relevance, since all 2D maps achieved the cluster data of a milk condition. Therefore, it can be concluded that 2D maps can also be an alternative to detect milk adulteration in these experimental setup conditions. 


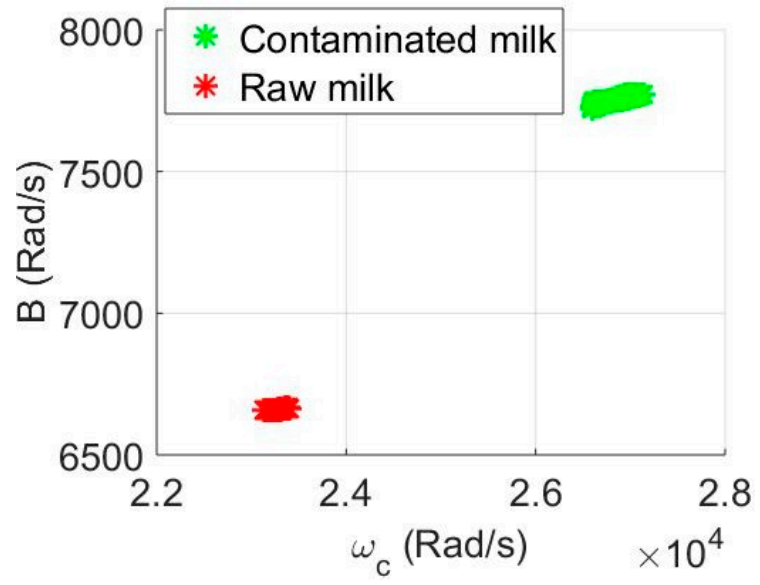

(a)

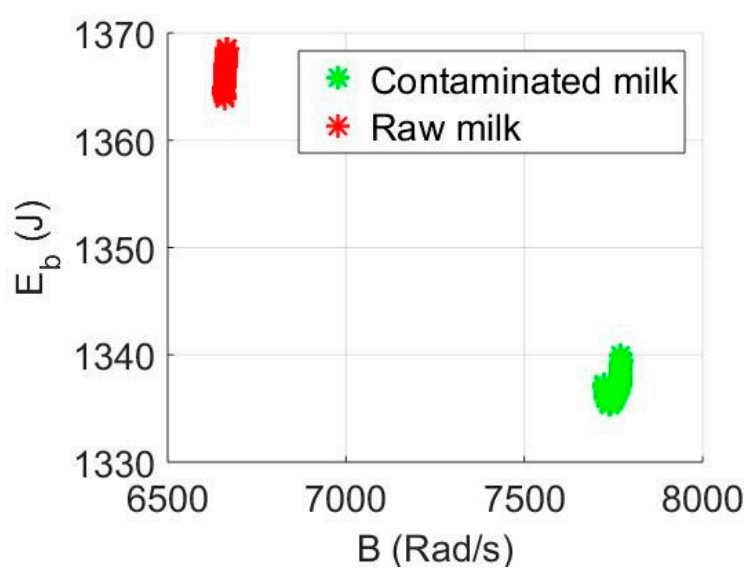

(b)

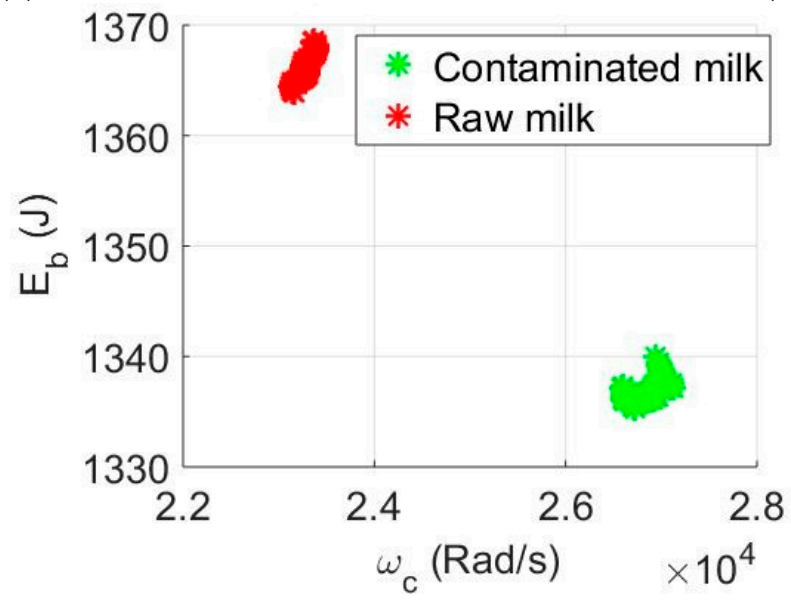

(c)

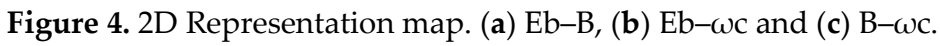

\section{Conclusions}

Sensors applied in the milk quality control are an important tool to ensure better quality for consumers. In this study, a pair of low-cost piezoelectric diaphragms were used in order to contribute to a methodology providing cheaper and faster evaluation of milk quality. The chromatic technique application was able to differentiate the raw milk from the milk with $1 \%$ sodium hydroxide using the data of the characteristics of mechanical acoustic propagation in the different samples, which were generated and acquired by the pair of emitter and receiver of piezoelectric diaphragms. Therefore, the results reported in this study indicate that low-cost piezoelectric diaphragms are promising for liquids quality control. From this work, some possibilities for future research appears, such as:

- Verifying whether the method identifies different variations in the concentration of contamination;

- Investigating if the sensor can differentiate more common forms of milk contamination such as with deionized water, hydrogen peroxide and formaldehyde;

- Applying the methodology to identify adulteration in other liquids such as gasoline, diesel and alcohol.

Author Contributions: All authors contributed in writing, proofreading, and providing suggestions for the improvement of the paper.

Conflicts of Interest: The authors declare no conflict of interest. 


\section{References}

1. Afzal, A.; Mahmood, M.S.; Hussain, I.; Akhtar, M. Adulteration and Microbiological Quality of Milk (A Review). Pak. J. Nutr. 2011, 1196, 184-203.

2. Durante, G.; Becari, W.; Lima, F.A.; Peres, H.E. Electrical Impedance Sensor for Real-Time Detection of Bovine Milk Adulteration. IEEE Sens. J. 2016, 16, 861-865.

3. Mabrook, M.F.; Petty, M.C. A novel technique for the detection of added water to full fat milk using single frequency admittance measurements. Sci. Direct Sens. Actuators, 2016, 96, 215-218.

4. Wang, X.; Li, X.; Rong, M.; Xie, D.; Ding, D.; Wang, Z. UHF signal processing and pattern recognition of partial discharge in gas-insulated switchgear using chromatic methodology. Sensors 2017, 17, 177, doi:10.3390/s17010177.

5. De Castro, B.A.; de Melo Brunini, D.; Baptista, F.G.; Andreoli, A.L.; Ulson, J.A. Assessment of macro fiber composite sensors for measurement of acoustic partial discharge signals in power transformers. IEEE Sens. J. 2017, 17, 6090-6099.

6. Castro, B.A.; Clerice, G.A.; Andreoli, A.L.; de Souza Campos, F.; Ulson, J.A. A low cost system for acoustic monitoring of partial discharge in power transformer by Piezoelectric Sensor. IEEE Lat. Am. Trans. 2016, 7, 3225-3231.

7. De Freitas, E.S.; Baptista, F.G.; Budoya, D.E.; de Castro, B.A. Equivalent circuit of piezoelectric diaphragms for impedance-based structural health monitoring applications. IEEE Sens. J. 2017, 17, 5537-5546.

8. Meitzler, A.H. IEEE Standard on Piezoelectricity: An American National Standard; IEEE-ANSI: New York, NY, USA, 1988; 66p.

9. Budoya, D.; Castro, B.D.; Campeiro, L.; Silveira, R.D.; Freitas, E.D.; Baptista, F. Analysis of Piezoelectric Diaphragms in Impedance-Based Damage Detection in Large Structures. Proceedings 2018, 2, 131.

10. Piezoelectric Diaphragms. Available online: https://www.murata.com/en-us/products/sound/diaphragm (accessed on 11 October 2018).

11. Singh, V.R.; Dwivedi, S. Ultrasonic Detection of Adulteration in Fluid Foods. In Proceedings of the RC IEEE-EMBS, New Delhi, India, 15-18 February 1995.

12. Castro, B.A.; Baptista, F.G.; Ciampa, F. Impedance-based Structural Health Monitoring Under Low Signalto-Noise Ratio Conditions. In Proceedings of the 9th European Workshop on Structural Health Monitoring, Manchester, UK, 10-13 July 2018; pp. 1-12.

13. Jones, G.R.; Russell, P.C.; Vourdas, A.; Cosgrave, J.; Stergioulas, L.; Haber, R. The Gabor transform basis of chromatic monitoring. Meas. Sci. Technol. 2000, 11, 489-498, doi:10.1088/0957-0233/11/5/307.

14. Ardila-Rey, J.A.; Montaña, J.; de Castro, B.A.; Schurch, R.; Covolan Ulson, J.A.; Muhammad-Sukki, F.; Bani, N.A. A Comparison of Inductive Sensors in the Characterization of Partial Discharges and Electrical Noise Using the Chromatic Technique. Sensors 2018, 18, 1021, doi:10.3390/s18041021.

15. Cheng, R.S.; Verdu, S. Capacity of root-mean-square bandlimited Gaussian multiuser channels. IEEE Trans. Inf. Theory 1991, 37, 453-465, doi:10.1109/18.79901.

(C) 2018 by the authors. Licensee MDPI, Basel, Switzerland. This article is an open access article distributed under the terms and conditions of the Creative Commons Attribution (CC BY) license (http://creativecommons.org/licenses/by/4.0/). 